\title{
SPECTRAL ANALYSIS OF SCHRÖDINGER OPERATORS WITH UNUSUAL SEMICLASSICAL BEHAVIOR
}

\author{
PAVEl Exner ${ }^{a, b}$, DiAna BARSEGhyan ${ }^{a, b, *}$ \\ ${ }^{a}$ Doppler Institute for Mathematical Physics and Applied Mathematics, Břehová 7, 11519 Prague \\ ${ }^{b}$ Nuclear Physics Institute ASCR, 25068 Řež near Prague, Czechia \\ * corresponding author: dianabar@ujf.cas.cz
}

\begin{abstract}
In this paper we discuss several examples of Schrödinger operators describing a particle confined to a region with thin cusp-shaped 'channels', given either by a potential or by a Dirichlet boundary; we focus on cases when the allowed phase space is infinite but the operator still has a discrete spectrum. First we analyze two-dimensional operators with the potential $|x y|^{p}-\lambda\left(x^{2}+y^{2}\right)^{p /(p+2)}$ where $p \geq 1$ and $\lambda \geq 0$. We show that there is a critical value of $\lambda$ such that the spectrum for $\lambda<\lambda_{\text {crit }}$ is below bounded and purely discrete, while for $\lambda>\lambda_{\text {crit }}$ it is unbounded from below. In the subcritical case we prove upper and lower bounds for the eigenvalue sums. The second part of work is devoted to estimates of eigenvalue moments for Dirichlet Laplacians and Schrödinger operators in regions having infinite cusps which are geometrically nontrivial being either curved or twisted; we are going to show how these geometric properties enter the eigenvalue bounds.
\end{abstract}

KEYWORDS: Schrödinger operator, discrete spectrum, Lieb-Thirring inequality, cusp-shaped regions, geometrically induced spectrum.

\section{INTRODUCTION}

The semiclassical method for analyzing the operator has proved itself a tremendously useful tool over the century since it was proposed by Hermann Weyl. Nevertheless, there are cases when estimates based on phase-space volume fail; the classical example is due to B. Simon [1] and describes a two-dimensional Schrödinger operator with the potential $|x y|^{p}$ having deep 'channels' the width of which is shrinking with the distance from the origin.

The present paper is devoted to a discussion of several models of this type. It summarizes the presentation of the second named author at the conference Analytic and algebraic methods in physics $X$ (Prague, 2012) based on the original papers [3, 4] to which we refer for details of the proofs which are sketched here.

Our first aim is to show that the effects known from the paper [1] can occur even if the potential is unbounded from below; at the same time the model will exhibit a parameter transition between different spectral regimes. One has to add that the first person to draw attention to the possibility of finding a discrete and below bounded spectrum in a below unbounded potential was to our knowledge M. Znojil, who analyzed a related model in [2]. In the second part we will discuss Schödinger operators and Dirichlet Laplacians on cusp-shaped regions which are geometrically nontrivial, being either bent or twisted, and show how their geometry is reflected in spectral properties.

\section{A MOdel With POTENTIAL Unbounded FROM BELOW AND INFINITE PhaSe SPACE}

We are going to consider here the following class of operators,

$$
L_{p}(\lambda): L_{p}(\lambda) \psi=-\Delta \psi+\left(|x y|^{p}-\lambda\left(x^{2}+y^{2}\right)^{p /(p+2)}\right) \psi, \quad p \geq 1
$$

on $L^{2}\left(\mathbb{R}^{2}\right)$ where $x, y$ are Cartesian coordinates in $\mathbb{R}^{2}$. The parameter $\lambda$ in the second term of the potential is assumed to be non-negative; unless its value is important in a particular context we write simply $L_{p}$. Since $\frac{2 p}{p+2}<2$ the operator 2.1 is e.s.a. on $C_{0}^{\infty}\left(\mathbb{R}^{2}\right)$ by the Faris-Lavine theorem - see [5, Theorems X.28, X.38]; in the following we mean by the symbol $L_{p}$ or $L_{p}(\lambda)$ always its closure.

We are going to demonstrate the existence of a critical value of the coupling constant $\lambda$, expressed explicitly in terms of the ground-state eigenvalue of the corresponding (an)harmonic oscillator Hamiltonian, such that the spectrum of $L_{p}(\lambda)$ is below bounded and purely discrete for $\lambda<\lambda_{\text {crit }}$, while for $\lambda>\lambda_{\text {crit }}$ it becomes unbounded from below. In the subcritical case we shall present upper and lower bounds to the sums of the first $N$ eigenvalues of $L_{p}(\lambda)$. 


\subsection{DisCRETENESS OF THE SPECTRUM}

Let us first look at small values of $\lambda$. To speak quantitatively we need an auxiliary operator which will be an (an)harmonic oscillator Hamiltonian $\tilde{H}_{p}: \tilde{H}_{p} u=-u^{\prime \prime}+|t|^{p} u$ on the natural domain in $L^{2}(\mathbb{R})$. Let $\gamma_{p}$ be the minimal eigenvalue of this operator; in view of the mirror symmetry we have $\gamma_{p}=\inf \sigma\left(H_{p}\right)$, where

$$
H_{p}: H_{p} u=-u^{\prime \prime}+t^{p} u
$$

on the natural domain in $L^{2}\left(\mathbb{R}_{+}\right)$with the Neumann condition at the origin. It is well known that the quantity $\gamma_{p}$ depends smoothly on $p$ being equal to one for $p=2$ and tending to $\gamma_{\infty}=\frac{1}{4} \pi^{2}$ as $p \rightarrow \infty$; a numerical analysis performed in [3] shows that the function $p \mapsto \gamma_{p}$ is convex and $\gamma_{p}>0.99$ for any $p \geq 1$.

Theorem 2.1. For any $\lambda \in\left[0, \lambda_{\text {crit }}\right)$, where $\lambda_{\text {crit }}:=\gamma_{p}$, the operator $L_{p}(\lambda)$ with $p \geq 1$ is bounded from below and its spectrum is purely discrete.

Sketch of the proof. Fix first $\lambda<\gamma_{p}$. By the minimax principle we need to estimate $L_{p}$ from below by a self-adjoint operator with a purely discrete spectrum. To this aim we employ a suitable bracketing imposing additional Neumann conditions at concentric circles of radii $n=1,2, \ldots$ Using the polar coordinates, we get a direct sum of operators acting as

$$
L_{n, p}^{(1)} \psi=-\frac{1}{r} \frac{\partial}{\partial r}\left(r \frac{\partial \psi}{\partial r}\right)-\frac{1}{n^{2}} \frac{\partial^{2} \psi}{\partial \varphi^{2}}+\left(\frac{r^{2 p}}{2^{p}}|\sin 2 \varphi|^{p}-\lambda r^{2 p /(p+2)}\right) \psi,\left.\quad \frac{\partial \psi}{\partial n}\right|_{r=n-1}=\left.\frac{\partial \psi}{\partial n}\right|_{r=n}=0
$$

on the regions $G_{n}:=\{(r, \varphi): n-1 \leq r<n, 0 \leq \varphi<2 \pi\}, n=1,2, \ldots$ Each of these annuli is compact and the potential is regular on it, hence $\sigma\left(L_{n, p}^{(1)}\right)$ is purely discrete. It thus suffices to check that inf $\sigma\left(L_{n, p}^{(1)}\right) \rightarrow \infty$ as $n \rightarrow \infty$, because the spectrum of $\bigoplus_{n=1}^{\infty} L_{n, p}^{(1)}$ below any fixed value will then be purely discrete. We estimate $L_{n, p}^{(1)}$ from below by an operator with separated variables,

$$
L_{n, p}^{(2)} \psi=-\frac{1}{r} \frac{\partial}{\partial r}\left(r \frac{\partial \psi}{\partial r}\right)-\frac{1}{n^{2}} \frac{\partial^{2} \psi}{\partial \varphi^{2}}+\left(\frac{(n-1)^{2 p}}{2^{p}}|\sin 2 \varphi|^{p}-\lambda n^{2 p /(p+2)}\right) \psi,\left.\quad \frac{\partial \psi}{\partial n}\right|_{r=n-1}=\left.\frac{\partial \psi}{\partial n}\right|_{r=n}=0,
$$

and establish that $\inf \sigma\left(L_{n, p}^{(3)}\right) \rightarrow \infty$ as $n \rightarrow \infty$, where $L_{n, p}^{(3)}$ is the angular part of $L_{n, p}^{(2)}$. The spectrum of $L_{n, p}^{(2)}$ is the 'sum' of the radial and angular component and the lowest radial eigenvalue is zero corresponding to a constant eigenfunction. One the other hand, the angular part behaves as the anharmonic oscillator around the potential minima, and the corresponding eigenvalue prevails over the negative $\lambda$-dependent term [3]. In this way one gets inf $\sigma\left(L_{n, p}^{(2)}\right) \rightarrow \infty$ as $n \rightarrow \infty$, which proves by the minimax principle the same for operator $L_{n, p}^{(1)}$.

\subsection{The SUPERCRITICAL CASE}

For large $\lambda$ the spectral behaviour is different.

Theorem 2.2. $\sigma\left(L_{p}(\lambda)\right), p \geq 1$, is unbounded from below if $\lambda>\lambda_{\text {crit }}$.

Sketch of the proof. We use a similar technique, this time looking for an upper bound to $L_{p}(\lambda)$ obtained by Dirichlet bracketing: we consider the operators $\widetilde{L}_{n, p}^{(1)}$ acting as 2.3 on the annular domains $G_{n}$ with Dirichlet boundary conditions. The latter give a contribution of order $\mathcal{O}(1)$ as $n \rightarrow \infty$ and since the $\lambda$-dependent term now prevails we conclude [3] that

$$
\inf \sigma\left(\widetilde{L}_{n, p}^{(1)}\right) \rightarrow-\infty \text { as } n \rightarrow \infty
$$

which means that $\sigma\left(L_{p}(\lambda)\right)$ is unbounded from below.

\subsection{LOWER BOUNDS TO EIGENVALUE SUMS}

Next we will show how the eigenvalue sums of operator $L_{p}(\lambda)$ can be estimated for small values of $\lambda$. We introduce the number

$$
\alpha:=\frac{1}{40}(5+\sqrt{105})^{2} \approx 5.81
$$

it is clear that $\alpha^{-1}<\gamma_{p}$. We denote by $\left\{\lambda_{j, p}\right\}_{j=1}^{\infty}$ the eigenvalues of $L_{p}(\lambda)$ arranged in ascending order; then we have the following result.

Theorem 2.3. To any nonnegative $\lambda<\alpha^{-1} \approx 0.172$ there is a positive $C_{p}$ depending on $p$ only such that

$$
\sum_{j=1}^{N} \lambda_{j, p} \geq C_{p}(1-\alpha \lambda) \frac{N^{(2 p+1) /(p+1)}}{\left(\ln ^{p} N+1\right)^{1 /(p+1)}}-c \lambda N, \quad N=1,2, \ldots
$$

where $c=2\left(\frac{\alpha^{2}}{5}+1\right) \approx 15.51$. 
Sketch of the proof. We denote by $\left\{\psi_{j, p}\right\}_{j=1}^{\infty}$ the system of normalized eigenfunctions corresponding to $\left\{\lambda_{j, p}\right\}_{j=1}^{\infty}$,

$$
-\Delta \psi_{j, p}+\left(|x y|^{p}-\lambda\left(x^{2}+y^{2}\right)^{p) /(p+2)}\right) \psi_{j, p}=\lambda_{j, p} \psi_{j, p}, \quad j=1,2, \ldots ;
$$

without loss of generality we may assume these functions to be real-valued. Our potential form hyperbolic-shaped 'channels' and we have first to estimate eigenfunction integrals in the corresponding parts of the plane. We establish [3] that for any natural $j$ and a $\delta>0$ one has

$$
\begin{aligned}
\int_{1}^{\infty} \int_{0}^{(1+\delta) y^{-p /(p+2)}} y^{2 p /(p+2)} \psi_{j, p}^{2}(x, y) \mathrm{d} x \mathrm{~d} y \\
\leq \frac{5}{2}(1+\delta)^{2} \int_{1}^{\infty} \int_{0}^{\infty}\left(\frac{\partial \psi_{j, p}}{\partial x}\right)^{2}(x, y) \mathrm{d} x \mathrm{~d} y+2 \frac{1+\delta}{\delta} \int_{1}^{\infty} \int_{0}^{(1+\delta) y^{-p /(p+2)}} x^{p} y^{p} \psi_{j, p}^{2}(x, y) \mathrm{d} x \mathrm{~d} y
\end{aligned}
$$

and that for an arbitrary $\varepsilon>0$ there is a number $\theta(\varepsilon) \in[1,1+\delta]$ such that

$$
\int_{1}^{\infty} y^{p /(p+2)} \psi_{j, p}^{2}\left(\frac{\theta(\varepsilon)}{y^{p /(p+2)}}, y\right) \mathrm{d} y<\frac{1}{\delta} \int_{1}^{\infty} \int_{y^{-p /(p+2)}}^{(1+\delta) y^{-p /(p+2)}} x^{p} y^{p} \psi_{j, p}^{2}(x, y) \mathrm{d} x \mathrm{~d} y+\varepsilon .
$$

Using the potential symmetry we get an analogous bound for the other 'channels'. Using next the normalization $\left\|\psi_{j, p}\right\|=1$ and the mentioned estimates we find

$$
\begin{gathered}
\int_{\mathbb{R}^{2}}\left(x^{2}+y^{2}\right)^{\frac{p}{p+2}} \psi_{j, p}^{2}(x, y) \mathrm{d} x \mathrm{~d} y \leq\left(\int_{|y| \geq 1} \int_{|x| \leq(1+\delta)|y|^{-p /(p+2)}}|y|^{2 p /(p+2)} \psi_{j, p}^{2}(x, y) \mathrm{d} x \mathrm{~d} y\right. \\
+\int_{|y| \geq 1} \int_{|x|>(1+\delta)|y|^{-p /(p+2)}}|y|^{2 p /(p+2)} \psi_{j, p}^{2}(x, y) \mathrm{d} x \mathrm{~d} y+\int_{|x| \geq 1} \int_{|y| \leq(1+\delta)|x|^{-p /(p+2)}}|x|^{2 p /(p+2)} \psi_{j, p}^{2}(x, y) \mathrm{d} y \mathrm{~d} x \\
\left.+\int_{|x| \geq 1} \int_{(1+\delta)|x|^{-p /(p+2)<|y|<1}}|x|^{2 p /(p+2)} \psi_{j, p}^{2}(x, y) \mathrm{d} y \mathrm{~d} x\right)+2 \\
\leq(1+\delta) \max \\
\left\{\frac{5}{2}(1+\delta), \frac{2}{\delta}\right\}\left(\int_{\mathbb{R}^{2}}\left|\nabla \psi_{j, p}\right|^{2}(x, y) \mathrm{d} x \mathrm{~d} y+\int_{\mathbb{R}^{2}}|x y|^{p} \psi_{j, p}^{2}(x, y) \mathrm{d} x \mathrm{~d} y+(1+\delta)^{2}\right)+2
\end{gathered}
$$

where we have used the inequality $|x y|^{p}>|y|^{2 p /(p+2)}$ valid on the domain of the second of the four integrals and an analogous bound for the fourth integral; the factor $(1+\delta)^{2}$ prevents double counting the 'corner regions' with $|x|,|y| \geq 1$ and $|y| \leq(1+\delta)|x|^{-p /(p+2)}$. Choosing $\delta=\frac{-5+\sqrt{105}}{10}$ we arrive at

$$
\int_{\mathbb{R}^{2}}\left(x^{2}+y^{2}\right)^{\frac{p}{p+2}} \psi_{j, p}^{2}(x, y) \mathrm{d} x \mathrm{~d} y \leq \alpha\left(\int_{\mathbb{R}^{2}}\left|\nabla \psi_{j, p}\right|^{2}(x, y) \mathrm{d} x \mathrm{~d} y+\int_{\mathbb{R}^{2}}|x y|^{p} \psi_{j, p}^{2}(x, y) \mathrm{d} x \mathrm{~d} y\right)+c,
$$

where $c:=\alpha(1+\delta)^{2}+2=2\left(\frac{\alpha^{2}}{5}+1\right)$. Since $\lambda_{j, p}$ is the eigenvalue corresponding to the eigenfunction $\psi_{j, p}$ the inequality derived above implies

$$
\int_{\mathbb{R}^{2}}\left|\nabla \psi_{j, p}\right|^{2} \mathrm{~d} x \mathrm{~d} y+\int_{\mathbb{R}^{2}}|x y|^{p} \psi_{j, p}^{2} \mathrm{~d} x \mathrm{~d} y \leq \frac{1}{1-\alpha \lambda}\left(\lambda_{j, p}+c \lambda\right), \quad j=1,2, \ldots
$$

Next we use Plancherel's theorem to express the gradients of $\psi_{j, p}$ in the first integral and then apply the following Lieb-Thirring-type inequality to the resulting orthonormal series:

Lemma 2.4. There is a constant $C_{p}^{\prime}$ such that for any orthonormal system of real-valued function, $\Phi=$ $\left\{\varphi_{j}\right\}_{j=1}^{N} \subset L^{2}\left(\mathbb{R}^{2}\right), N=1,2, \ldots$, the inequality

$$
\int_{\mathbb{R}^{2}} \rho_{\Phi}^{p+1} \mathrm{~d} x \mathrm{~d} y \leq C_{p}^{\prime}\left(\ln ^{p} N+1\right) \sum_{j=1}^{N} \int_{\mathbb{R}^{2}}|\xi \eta|^{p}\left|\hat{\varphi}_{j}\right|^{2} \mathrm{~d} \xi \mathrm{d} \eta
$$

holds true, where $\rho_{\Phi}:=\sum_{j=1}^{N} \varphi_{j}^{2}$.

This claim was proved as Theorem 2 in [6] for $p=1$ and it is straightforward to extend the argument to any $p \geq 1$. After a few simple manipulations for any non-negative parameter $\varrho$ we get

$$
C_{p}^{\prime \prime}\left(1+\ln ^{p} N\right)^{1 / p} \varrho^{(2 p+1) / p} \geq N \varrho-\frac{1}{1-\alpha \lambda} \sum_{j=1}^{N}\left(\lambda_{j, p}+c \lambda\right)
$$


with a new positive constant $C_{p}^{\prime \prime}$.

In the final step we consider

$$
\widetilde{g}(N)=\max _{\varrho \geq 0}\left(N \varrho-C_{p}^{\prime \prime} \varrho^{(2 p+1) / p}\left(1+\ln ^{p} N\right)^{1 / p}\right) .
$$

Denoting now the expression in the bracket as $h(\varrho)$ we check easily that reaches its maximum at the point

$$
\varrho_{\max }=\left(p /(2 p+1) C_{p}^{\prime \prime}\right)^{p /(p+1)} N^{p /(p+1)}\left(1+\ln ^{p} N\right)^{-1 /(p+1)}
$$

and its value there equals

$$
\widetilde{g}(N)=h\left(\varrho_{\max }\right)=C_{p} \frac{N^{(2 p+1) /(p+1)}}{\left(1+\ln ^{p} N\right)^{1 /(p+1)}}
$$

with some constant $C_{p}>0$. In this way we arrive at the bound

$$
\frac{1}{1-\alpha_{p} \lambda} \sum_{j=1}^{N}\left(\lambda_{j, p}+c_{p} \lambda\right) \geq h\left(\varrho_{\max }\right)=\widetilde{g}(N) .
$$

which is equivalent to the claim of the theorem.

\subsection{UPPER BOUNDS}

Next we estimate spectral sums of $L_{p}(\lambda), p \geq 1$, from above for any subcritical $\lambda$. It will show, in particular, that in the case $\lambda=0$ the asymptotics given by Theorem 2.3 is exact up the value of the constant.

Theorem 2.5. To any $p \geq 1$ there is a constant $\widetilde{C}_{p}>0$ such that

$$
\sum_{j=1}^{N} \lambda_{j, p} \leq \widetilde{C}_{p} \frac{N^{(2 p+1) /(p+1)}}{\left(1+\ln ^{p} N\right)^{1 /(p+1)}}, \quad N=1,2, \ldots
$$

holds for any $0 \leq \lambda<\gamma_{p}$.

Sketch of the proof. It clearly suffices to prove the claim for $\lambda=0$. Consider the operator $\hat{H}_{p}=-\Delta+Q$, where $Q(x, y)=|x y|^{p}+|x|^{p}+|y|^{p}+1$ in $L^{2}\left(\mathbb{R}^{2}\right)$. Its spectrum is discrete by Theorem 2.1 in combination with the minimax principle since $H_{p} \geq L_{p}$, thus we have to establish the bound of the indicated type for the eigenvalues $0 \leq \beta_{1, p} \leq \beta_{2, p} \leq \cdots$ of the estimating operator $\hat{H}_{p}$.

We employ Weyl asymptotics for the number of eigenvalues of below-bounded differential operators in a version due to $\mathrm{G}$. Rozenblum. Let $T=-\Delta+V$ in $\mathbb{R}^{m}$, where the potential $V(x) \geq 1$ and tends to infinity as $|x| \rightarrow \infty$. We denote by $E(\lambda, V)$ the set $\left\{x \in \mathbb{R}^{m}: V(x)<\lambda\right\}$ and put

$$
\sigma(\lambda, V)=\operatorname{mes} E(\lambda, V) .
$$

For any unit cube $D \subset \mathbb{R}^{m}$ we denote the mean value of the function $V$ in $D$ by $V_{D}$. Furthermore, given a function $f \in L^{1}(D)$ and $t \leq \sqrt{m}$ we define its $L_{1}$-modulus of continuity by the formula

$$
\omega_{1}(f, t, D):=\sup _{|z|<t} \int_{x \in D, x+z \in D}|f(x+z)-f(x)| \mathrm{d} x .
$$

Then we have the following result [7]:

Lemma 2.6. Suppose that the potential $V$ satisfies the conditions:

(1.) There is $c>0$ such that $\sigma(2 \lambda, V) \leq c \sigma(\lambda, V)$ holds all $\lambda$ large enough.

(2.) $V(y) \leq c V(x)$ holds if as $|x-y| \leq 1$.

(3.) There is a continuous and monotonous function $\eta: t \in[0, \sqrt{m}] \rightarrow \mathbb{R}_{+}$with $\eta(0)=0$ and a number $\beta \in\left[0, \frac{1}{2}\right)$ such that for any unit square $D$ we have

$$
\omega_{1}(V, t, D) \leq \eta(t) t^{2 \beta} V_{D}^{1+\beta}
$$


Under these assumptions the asymptotic formula

$$
N(\lambda, V) \sim \Gamma_{m} \Phi(\lambda, V)
$$

holds for the operator $T=-\triangle+V$, where $N(\lambda, V)$ is the number of eigenvalues of $T$ smaller than $\lambda$, unit-ball volume $\Gamma_{m}=(2 \sqrt{\pi})^{-m}\left(\Gamma\left(\frac{m}{2}+1\right)\right)^{-1}$, and

$$
\Phi(\lambda, V)=\int_{\mathbb{R}^{2}}(\lambda-V)_{+}^{m / 2} \mathrm{~d} x \mathrm{~d} y .
$$

It is straightforward to check the hypotheses of the lemma for the operator $\hat{H}_{p}$, which gives an asymptotic formula for spectral distribution function $N\left(\lambda, \hat{H}_{p}\right)$. After some simple estimates [3] we arrive at the following upper bound on the spectrum of the operator $\hat{H}_{p}$,

$$
\sum_{j=1}^{N} \beta_{j, p} \leq \widetilde{C_{p}} \frac{N^{(2 p+1) /(p+1)}}{(\ln N+1)^{p /(p+1)}}
$$

with a constant $\widetilde{C_{p}}$ depending on $p$ only; this proves the theorem.

\section{SCHRÖDINGER OPERATORS IN CUSPS WITH NON-TRIVIAL GEOMETRY}

In the second part of the paper we shall discuss Schrödinger type operators

$$
H_{\Omega}=-\Delta_{D}^{\Omega}-V
$$

with a bounded measurable potential $V \geq 0$ on $L^{2}(\Omega)$, where $-\Delta_{D}^{\Omega}$ is the Dirichlet Laplacian on a region $\Omega \subset \mathbb{R}^{d}$. In the spirit of the previous considerations we will be particularly interested in situations where $\Omega$ is unbounded but $H_{\Omega}$ still has a purely discrete spectrum. It is well known [1, 9] that for some unbounded regions the spectrum may be purely discrete; typically this happens if $\Omega$ has cusps. The negative spectrum of $H_{\Omega}$ consists of a finite number of eigenvalues counted with their multiplicities; it is natural to ask about bounds on the negative spectrum moments in terms of their geometrical properties, in the spirit the seminal work of Lieb and Thirring [10, or in the present context referring to Berezin, Lieb, Li and Yau [11 14]. Note that estimates of this type have been derived recently in [5] for various cusped regions; a typical example is $\Omega=\left\{(x, y) \in \mathbb{R}^{2}:|x y|<1\right\}$ with hyperbolic ends. Our goal is to discuss situations when such infinite cusps of $\Omega$ are geometrically nontrivial, being curved or twisted, to see in which way the geometry influences the spectral estimates.

First we will discuss a curved planar cusp and derive estimates on negative spectrum moments which include a curvature-induced potential. This result can be generalized for bent cusps with a circular cross section; we refer to [4] for a description. Then, in Section 3.2, we will consider cusps of a non-circular cross section in $\mathbb{R}^{3}$ which are straight but twisted. The geometry of the region will again be involved in the obtained eigenvalue estimates, now in a different way than for curved cusps, because the effective interaction associated with twisting is repulsive rather than attractive.

\subsection{Curved Planar Cusps}

We consider an infinite cusp-shaped $\Omega \subset \mathbb{R}^{2}$ assuming that its boundary is smooth, so one can describe it by specifying its axis and the cusp width at each point of it. This will allow us to employ natural curvilinear coordinates by analogy with the theory of quantum waveguides [15] and to 'straighten' the cusp, translating its geometric properties into the coefficients of the resulting operator.

To be specific, we characterize our region by three functions: sufficiently smooth $a, b: \mathbb{R} \rightarrow \mathbb{R}^{2}$ and a positive and continuous $f: \mathbb{R} \rightarrow \mathbb{R}^{+}$in such a way that

$$
\Omega:=\{(a(s)-u \dot{b}(s), b(s)+u \dot{a}(s)): s \in \mathbb{R},|u|<f(s)\},
$$

where the dot marks the derivative with respect to $s$; to make the region $\Omega$ cusp-shaped we shall always suppose that

$$
\lim _{|s| \rightarrow \infty} f(s)=0
$$

Since the reference curve $\Gamma=\{(a(s), b(s)): s \in \mathbb{R}\}$ can always be parametrized by its arc length we may suppose without loss of generality that $\dot{a}(s)^{2}+\dot{b}(s)^{2}=1$ and $s$ is the arc length. The signed curvature $\gamma(s)$ of $\Gamma$ is then given by

$$
\gamma(s)=\dot{b}(s) \ddot{a}(s)-\dot{a}(s) \ddot{b}(s)
$$


knowing this one can reconstruct the functions $a, b$ describing the Cartesian coordinates of the cusps axis, modulo Euclidean transformations.

Under the condition (3.3) the region is quasi-bounded so it may have a purely discrete spectrum. This is indeed the case; recall that the necessary and sufficient condition for the purely discrete spectral character [16. Thm 2.8] is that we can cover $\Omega$ by a family of unit balls whose centres tend to infinity in such a way that the volumes of their intersections with $\Omega$ tend to zero; it is not difficult to construct such a ball sequence if $(3.3)$ is valid.

Our main aim is to provide bounds on the eigenvalue moments; as usual when dealing with a Lieb-Thirring type problem we restrict our attention to the negative part of the spectrum noting that it can be always made non-empty by including a suitable constant into the potential.

Theorem 3.1. Consider the Schrödinger operator (3.1) on the region (3.2). Suppose that the curvature $\gamma \in C^{4}$, the inequality $\|f(\cdot) \gamma(\cdot)\|_{L^{\infty}(\mathbb{R})}<1$ holds true, and $\Omega$ does not intersect itself. Then for any $\sigma \geq 3 / 2$ we have the estimate

$$
\operatorname{tr}\left(H_{\Omega}\right)_{-}^{\sigma} \leq\|1+f|\gamma|\|_{\infty}^{-2 \sigma} L_{\sigma, 1}^{\mathrm{cl}} \int_{\mathbb{R}} \sum_{j=1}^{\infty}\left(-\left(\frac{\pi j}{2 f(s)}\right)^{2}+\|1+f|\gamma|\|_{\infty}^{2} W^{-}(s)+\|1+f|\gamma|\|_{\infty}^{2}\|\widetilde{V}(s, \cdot)\|_{\infty}\right)_{+}^{\sigma+1 / 2} \mathrm{~d} s
$$

where $\|\cdot\|_{\infty} \equiv\|\cdot\|_{L^{\infty}(\mathbb{R})}$ and $L_{\sigma, 1}^{\mathrm{cl}}$ is the semiclassical constant,

$$
L_{\sigma, 1}^{\mathrm{cl}}:=\frac{\Gamma(\sigma+1)}{\sqrt{4 \pi} \Gamma\left(\sigma+\frac{3}{2}\right)}
$$

and furthermore, we have introduced

$$
W^{-}(s):=\frac{\gamma(s)^{2}}{4(1-f(s)|\gamma(s)|)^{2}}+\frac{f(s)|\ddot{\gamma}(s)|}{2(1-f(s)|\gamma(s)|)^{3}}+\frac{5 f^{2}(s) \dot{\gamma}(s)^{2}}{4(1-f(s)|\gamma(s)|)^{4}}
$$

and $\widetilde{V}(s, u):=V(a(s)-u \dot{b}(s), b(s)+u \dot{a}(s))$.

Sketch of the proof. Using the mentioned 'straightening' transformation [15] we infer that $H_{\Omega}$ is unitarily equivalent to the operator $H_{0}$ on $L^{2}\left(\Omega_{0}\right)$ acting as

$$
\left(H_{0} \psi\right)(s, u)=-\frac{\partial}{\partial s}\left(\frac{1}{(1+u \gamma(s))^{2}} \frac{\partial \psi}{\partial s}(s, u)\right)-\frac{\partial^{2} \psi}{\partial u^{2}}(s, u)+((W-\widetilde{V}) \psi)(s, u),
$$

where $\Omega_{0}=\{(s, u): s \in \mathbb{R},|u|<f(s)\}$, the curvature-induced potential is

$$
W(s, u):=-\frac{\gamma^{2}(s)}{4(1+u \gamma(s))^{2}}+\frac{u \ddot{\gamma}(s)}{2(1+u \gamma(s))^{3}}-\frac{5}{4} \frac{u^{2} \dot{\gamma}^{2}(s)}{(1+u \gamma(s))^{4}}
$$

and Dirichlet boundary conditions are imposed at $u= \pm f(s)$. In view of the unitary equivalence it is enough to establish inequality 3.4 for the operator $H_{0}$. We employ the minimax principle: consider the operator $H_{0}^{-}$ defined on the domain $\mathcal{H}_{0}^{2}\left(\Omega_{0}\right)$ in $L^{2}\left(\Omega_{0}\right)$ by

$$
H_{0}^{-}=-\Delta_{D}^{\Omega_{0}}-\|1+f|\gamma|\|_{\infty}^{2}\left(W^{-}+\widetilde{V}\right),
$$

where $-\Delta_{D}^{\Omega_{0}}$ is as usual the corresponding Dirichlet Laplacian; it is obvious that

$$
H_{0} \geq\|1+f|\gamma|\|_{\infty}^{-2} H_{0}^{-}
$$

holds true, therefore it is sufficient to get the upper bound for negative eigenvalue moments of operator $H_{0}^{-}$.

We use a variational argument — see [17] — to estimate the negative eigenvalue moments of $H_{0}^{-}$by the negative eigenvalue moments of the other operator with the operator-valued potential defined on domain $\mathcal{H}^{1}\left(\mathbb{R}, L^{2}(\mathbb{R})\right)$ and given as follows

$$
-\frac{\partial^{2}}{\partial s^{2}} \otimes I_{L^{2}(\mathbb{R})}+H\left(s, \widetilde{V}, W^{-}\right)
$$

where $H\left(s, \widetilde{V}, W^{-}\right)$is the negative part of the Sturm-Liouville operator

$$
-\frac{\mathrm{d}^{2}}{\mathrm{~d} u^{2}}-\|1+f|\gamma|\|_{\infty}^{2}\left(W^{-}+\widetilde{V}\right) .
$$


Consequently,

$$
\operatorname{tr}\left(H_{0}^{-}\right)_{-}^{\sigma} \leq \operatorname{tr}\left(-\frac{\partial^{2}}{\partial s^{2}} \otimes I_{L^{2}(\mathbb{R})}+H\left(s, \tilde{V}, W^{-}\right)\right)_{-}^{\sigma}
$$

holds for any nonnegative number $\sigma$. This makes it possible to employ the version of Lieb-Thirring inequality for operator-valued potentials 18 for operator-valued potentials, which yields

$$
\operatorname{tr}\left(H_{0}^{-}\right)_{-}^{\sigma} \leq L_{\sigma, 1}^{\mathrm{cl}} \int_{\mathbb{R}} \operatorname{tr}\left(H\left(s, \widetilde{V}, W^{-}\right)\right)_{-}^{\sigma+1 / 2} \mathrm{~d} s, \quad \sigma \geq 3 / 2,
$$

with the semiclassical constant $L_{\sigma, 1}^{\mathrm{cl}}$. It remains to estimate the negative spectrum of the Sturm-Liouville operator $-\frac{\mathrm{d}^{2}}{\mathrm{~d} u^{2}}-\|1+f|\gamma|\|_{\infty}^{2}\left(W^{-}(s)+\|\widetilde{V}(s, \cdot)\|_{\infty}\right)$ which is easily done,

$$
\left(\left(\frac{\pi j}{2 f(s)}\right)^{2}-\|1+f|\gamma|\|_{\infty}^{2} W^{-}(s)-\|1+f|\gamma|\|_{\infty}^{2}\|\widetilde{V}(s, \cdot)\|_{\infty}\right)_{-}
$$

hence in view of $(3.6)$ and $(3.7)$ we find that

$$
\operatorname{tr}\left(H_{0}\right)_{-}^{\sigma} \leq\|1+f|\gamma|\|_{\infty}^{-2 \sigma} L_{\sigma, 1}^{\mathrm{cl}} \int_{\mathbb{R}} \sum_{j=1}^{\infty}\left(-\left(\frac{\pi j}{2 f(s)}\right)^{2}+\|1+f|\gamma|\|_{\infty}^{2} W^{-}(s)+\|1+f|\gamma|\|_{\infty}^{2}\|\widetilde{V}(s, \cdot)\|_{\infty}\right)_{+}^{\sigma+1 / 2} \mathrm{~d} s
$$

which proves the theorem.

We finish this section with two remarks. First we note that while the standard phase-space-volume estimates give correct high-energy behaviour one can find finite regions $\Omega$ for which there exists an intermediate energy region where the bound $(3.4)$ is much stronger than the Berezin-Li-Yau inequality. An example is given in paper [4], to which we refer also for the mentioned generalization of Theorem 3.1 to higher dimensions.

\subsection{TWisted CUSPS OF NON-CIRCULAR CROSS SECTION IN $\mathbb{R}^{3}$}

Let us now look at another type of nontrivial cusp geometry. As before we will suppose that its cross section changes along the curve playing role of the axis, however, now we allow it to be non-circular. Consider an open connected set $\omega_{0} \subset \mathbb{R}^{2}$ and a positive function $f: \mathbb{R} \rightarrow \mathbb{R}$ satisfying the condition (3.3), and set

$$
\omega_{s}:=f(s) \omega_{0},
$$

where we use the conventional shorthand $\alpha A:=\{(\alpha x, \alpha y):(x, y) \in A\}$ for $\alpha>0$ and $A \subset \mathbb{R}^{2}$. Using (3.8) we define a straight cusped region determined by $\omega_{0}$ and the function $f$ as $\Omega_{0}:=\left\{(s, x, y): s \in \mathbb{R},(x, y) \in \omega_{s}\right\}$.

Next we twist the region $\Omega_{0}$. We fix a $C^{1}$-smooth function $\theta: \mathbb{R} \rightarrow \mathbb{R}$ with a bounded derivative, $\|\dot{\theta}\|_{\infty}<\infty$, and introduce the set $\Omega_{\theta}$ as the image

$$
\Omega_{\theta}:=\mathfrak{L}_{\theta}\left(\Omega_{0}\right)
$$

where the map $\mathfrak{L}_{\theta}: \mathbb{R}^{3} \rightarrow \mathbb{R}^{3}$ is given by

$$
\mathfrak{L}_{\theta}(s, x, y):=(s, x \cos \theta(s)+y \sin \theta(s),-x \sin \theta(s)+y \cos \theta(s)) .
$$

We are interested primarily in nontrivial situations, assuming that

(1.) the function $\theta$ is not constant,

(2.) $\omega_{0}$ is not rotationally symmetric with respect to the origin in $\mathbb{R}^{2}$.

To formulate the result of this section, we need a few more preliminaries. First of all, we introduce $\varrho:=$ $\sup _{(x, y) \in \omega_{0}} \sqrt{x^{2}+y^{2}}$ and assume that

$$
\varrho\|f(\cdot) \dot{\theta}(\cdot)\|_{\infty}<1
$$

Next we set $\widetilde{V}(s, x, y):=V\left(\mathfrak{L}_{\theta}(s, x, y)\right)$ by analogy with the corresponding definitions in the previous sections, and finally, we introduce the operator

$$
L_{\text {trans }}:=-i\left(x \frac{\partial}{\partial y}-y \frac{\partial}{\partial x}\right), \quad \operatorname{Dom}\left(L_{\text {trans }}\right)=\mathcal{H}_{0}^{1}\left(\omega_{0}\right),
$$

describing the angular momentum component canonically associated with rotations in the transverse plane. We have the following claim: 
Theorem 3.2. Let $H_{\Omega_{\theta}}$ be the operator (3.1) referring to the region $\Omega_{\theta}$ defined by (3.9) and (3.10) with a potential $V \geq 0$ which is bounded and measurable. Under the assumption (3.11) for the negative spectrum of $H_{\Omega_{\theta}}$ the inequality

$$
\operatorname{tr}\left(H_{D}^{\Omega_{\theta}}\right)_{-}^{\sigma} \leq L_{\sigma, 1}^{\mathrm{cl}}\left(1-\varrho\|f \dot{\theta}\|_{\infty}\right)^{\sigma} \int_{\mathbb{R}} \sum_{j=1}^{\infty}\left(-\frac{\lambda_{0, j}(s)}{f^{2}(s)}+\frac{\|\widetilde{V}\|_{\infty}}{1-\varrho\|f \dot{\theta}\|_{\infty}}\right)_{+}^{\sigma+1 / 2} \mathrm{~d} s
$$

holds true for $\sigma \geq 3 / 2$, where $L_{\sigma, 1}^{\mathrm{cl}}$ is the constant 3.5 and $\lambda_{0, j}(s), j=1,2, \ldots$, are the eigenvalues of the operator

$$
H_{f, \theta}(s):=-\Delta_{D}^{\omega_{0}}+f^{2}(s) \dot{\theta}^{2}(s) L_{\text {trans }}^{2}
$$

defined on the domain $\mathcal{H}_{0}^{2}\left(\omega_{0}\right)$ in $L^{2}\left(\omega_{0}\right)$.

Sketch of the proof. As before, we employ suitable curvilinear coordinates, this time to 'untwist' the region. We define a unitary operator from $L^{2}\left(\Omega_{\theta}\right)$ to $L^{2}\left(\Omega_{0}\right)$ by $U_{\theta} \psi:=\psi \circ \mathfrak{L}_{\theta}$ which allows us to pass from $H_{\Omega_{\theta}}^{D}$ to the operator

$$
H_{0}:=U_{\theta}\left(H_{\Omega_{\theta}}^{D}\right) U_{\theta}^{-1}
$$

in $L^{2}\left(\Omega_{0}\right)$. From paper [19] we know that $H_{0}$ is the self-adjoint operator associated with the quadratic form

$$
Q_{0}: Q_{0}[\psi]:=\left\|\partial_{s} \psi+i \dot{\theta} L_{\mathrm{trans}} \psi\right\|^{2}+\left\|\nabla^{\prime} \psi\right\|^{2}-\int_{\Omega_{0}}\left(\widetilde{V}|\psi|^{2}\right)(s, x, y) \mathrm{d} s \mathrm{~d} x \mathrm{~d} y
$$

defined on $\mathcal{H}_{0}^{1}$, where $\nabla^{\prime}:=\left(\partial_{x}, \partial_{y}\right)$ is the transversal gradient and the norms refer to $L^{2}\left(\Omega_{0}\right)$. For any function $\psi \in H_{0}^{1}\left(\omega_{s}\right)$ we have

$$
\left|L_{\text {trans }} \psi\right| \leq \varrho f(s)\left|\nabla^{\prime} \psi\right|
$$

and applying the Cauchy-Schwarz inequality we infer

$$
2\left|\int_{\Omega_{0}} \dot{\theta} \partial_{s} \psi \overline{L_{\text {trans }} \psi} \mathrm{d} s \mathrm{~d} x \mathrm{~d} y\right| \leq \varrho\|f \dot{\theta}\|_{\infty}\left(\left\|\partial_{s} \psi\right\|_{L^{2}\left(\Omega_{0}\right)}^{2}+\left\|\nabla^{\prime} \psi\right\|_{L^{2}\left(\Omega_{0}\right)}^{2}\right),
$$

thus $Q_{0}[\psi]$ can be estimated from below by

$$
\left(1-\varrho\|f \dot{\theta}\|_{\infty}\right)\left(\|\nabla \psi\|_{L^{2}\left(\Omega_{0}\right)}^{2}+\left\|\dot{\theta} L_{\mathrm{trans}} \psi\right\|_{L^{2}\left(\Omega_{0}\right)}^{2}\right)-\int_{\Omega_{0}}\|\widetilde{V}(s, \cdot)\|_{\infty}|\psi|^{2} \mathrm{~d} s \mathrm{~d} x \mathrm{~d} y
$$

We introduce the operator

$$
H_{0}^{-}=-\Delta_{D}^{\Omega_{0}}+\dot{\theta}^{2} L_{\text {trans }}^{2}-\frac{1}{1-\varrho\|f \dot{\theta}\|_{\infty}}\|\widetilde{V}(s, \cdot)\|_{\infty}
$$

defined on $H_{0}^{2}\left(\omega_{0}\right)$, then the above estimate implies

$$
H_{0} \geq\left(1-\varrho\|f \dot{\theta}\|_{\infty}\right) H_{0}^{-}
$$

hence by the minimax principle and the condition 3.11 it is enough to establish the upper estimate for the negative spectrum of operator $H_{0}^{-}$.

Using again the variational technique one can estimate the negative eigenvalue moments of operator $H_{0}^{-}$ by the moments of the operator with separated variables, $-\frac{\partial^{2}}{\partial s^{2}} \otimes I_{L^{2}\left(\mathbb{R}^{2}\right)}+H(s, \widetilde{V})$, defined on the domain $H^{1}\left(\mathbb{R}, L^{2}\left(\mathbb{R}^{2}\right)\right)$, i.e.

$$
\operatorname{tr}\left(H_{0}^{-}\right)_{-}^{\sigma} \leq \operatorname{tr}\left(-\frac{\partial^{2}}{\partial s^{2}} \otimes I_{L^{2}\left(\mathbb{R}^{2}\right)}+H(s, \widetilde{V})\right)_{-}^{\sigma}, \quad \sigma \geq 0 .
$$

In view of the Lieb-Thirring inequality for operator valued potentials this implies

$$
\operatorname{tr}\left(H_{0}^{-}\right)_{-}^{\sigma} \leq L_{\sigma, 1}^{c l} \int_{\mathbb{R}} \operatorname{tr} H(s, \widetilde{V})_{-}^{\sigma+1 / 2} \mathrm{~d} s, \quad \text { for any } \quad \sigma \geq 3 / 2
$$

with the semiclassical constant $L_{\sigma, 1}^{c l}$. Then, by virtue of unitary equivalence of operators $H_{D}^{\Omega_{\theta}}$ and $H_{0}$, inequalities 3.12, 3.13 and the condition 3.11 we get

$$
\operatorname{tr}\left(H_{D}^{\Omega_{\theta}}\right)_{-}^{\sigma} \leq L_{\sigma, 1}^{\mathrm{cl}}\left(1-\varrho\|f \dot{\theta}\|_{\infty}\right)^{\sigma} \int_{\mathbb{R}} \operatorname{tr} H(s, \widetilde{V})_{-}^{\sigma+1 / 2} \mathrm{~d} s \quad \text { for } \quad \sigma \geq 3 / 2 .
$$


It is easy to see that the eigenvalues of operator $H(s, \widetilde{V})$ are

$$
\left(\frac{\lambda_{0, j}(s)}{f^{2}(s)}-\frac{1}{1-\varrho\|f \dot{\theta}\|_{\infty}}\|\widetilde{V}(s, \cdot)\|_{\infty}\right)_{-}, \quad j=1,2, \ldots,
$$

where $\lambda_{0, j}(s), j=1,2, \ldots$ are eigenvalues of the operator

$$
H_{f, \theta}(s):=-\Delta_{D}^{\omega_{0}}+f^{2}(s) \dot{\theta}^{2}(s) L_{\text {trans }}^{2} .
$$

From inequalities 3.14 and 3.15 it follows that $\operatorname{tr}\left(H_{D}^{\Omega_{\theta}}\right)_{-}^{\sigma}$ is estimated by

$$
L_{\sigma, 1}^{c l}\left(1-\varrho\|f \dot{\theta}\|_{\infty}\right)^{\sigma} \int_{\mathbb{R}} \sum_{j=1}^{\infty}\left(-\frac{\lambda_{0, j}(s)}{f^{2}(s)}+\frac{1}{1-\varrho\|f \dot{\theta}\|_{\infty}}\|\widetilde{V}(s, \cdot)\|_{\infty}\right)_{+}^{\sigma+1 / 2} \mathrm{~d} s
$$

for any $\sigma \geq 3 / 2$ which proves the theorem.

\section{ACKNOWLEDGEMENTS}

The research presented here was supported by the Czech Science Foundation within project P203/11/0701. The authors are grateful to the referees for reading the manuscript carefully and pointing out some minor slips of the pen.

\section{REFERENCES}

[1] Simon B.: Some quantum operators with discrete spectrum but classically continuous spectrum, Ann. Phys. 146 (1983), 209-220.

[2] Znojil M.: Quantum exotic: a repulsive and bottomless confining potential, J. Phys. A: Math. Gen. 31 (1998), 3349-3355.

[3] Exner P., Barseghyan D.: Spectral estimates for a class of Schrödinger operators with infinite phase space and potential unbounded from below, J. Phys. A: Math. Theor. 45 (2012), 075204.

[4] Exner P., Barseghyan D.: Spectral estimates for Dirichlet Laplacians and Schrödinger operators on geometrically nontrivial cusps, to appear in J. Spect. Theory, arXiv:1203.2098

[5] Reed M., Simon B.: Methods of Modern Mathematical Physics, II. Fourier Analysis. Self-Adjointness, Academic Press, New York 1975.

[6] Barseghyan D.: On the possibility of strengthening the Lieb-Thirring inequality, Math. Notes 86 (2009), 803-818.

[7] Rozenblum. G.: Asymptotics of the eigenvalues of Schrödinger operator, Mat. Sbornik (N.S.) 93(135) (1974), $347-367$.

[8] Adams R.A., Fournier J.F.: Sobolev spaces, 2nd ed., Academic Press, New York 2003.

[9] Geisinger L., Weidl T.: Sharp spectral estimates in domains of infinite volume, Rev. Math. Phys. 23 (2011), 615-641.

[10] Lieb E.H., Thirring W.: Inequalities for the moments of the eigenvalues of the Schrödinger Hamiltonian and their relation to Sobolev inequalities, in Studies in Math. Phys., Essays in Honor of Valentine Bargmann (E. Lieb, B. Simon and A.S. Wightman, eds.); Princeton Univ. Press, Princeton 1976; pp. 269-330.

[11] Berezin F.A.: Covariant and contravariant symbols of operators, Izv. Akad. Nauk SSSR Ser. Mat. 36 (1972), 1134-1167.

[12] Berezin F.A.: Convex functions of operators, Mat. Sb. (NS) 36(130) (1972), 268-276.

[13] Lieb E.H.: The classical limit of quantum spin systems, Commun. Math. Phys. 31 (1973), 327-340.

[14] Li P., Yau S.T.: On the Schrödinger equation and the eigenvalue problem, Commun. Math. Phys. 88 (1983), 309-318.

[15] Exner P., Šeba P.: Bound states in curved quantum wavequides, J. Math. Phys. 30 (1989), 2574-2580.

[16] Berger M.S., Schechter M.: Embedding theorems and quasi-linear elliptic boundary value problems for unbounded domain, Trans. Am. Math. Soc. 172 (1972), 261-278.

[17] Weidl T.: Improved Berezin-Li-Yau inequalities with a remainder term, in Spectral Theory of Differential Operators, Amer. Math. Soc. Transl. 225 (2008), 253-263.

[18] Laptev A., Weidl T.: Sharp Lieb-Thirring inequalities in high dimensions, Acta Math. 184 (2000), 87-100.

[19] Krejčiř́ík D., Zuazua E.: The Hardy inequality and the heat equation in the twisted tubes, J. Diff. Eqs. 250 (2011), $2334-2346$. 